\title{
VII Congreso de la Asociación de Historia Económica
}

Zaragoza, 19-20-21 de septiembre de 2001

\section{Conferencia inaugural:}

La historia de la peseta

Luis Ángel Rojo

Sesiones plenarias:

Economia del primer franquismo

Carlos Barciela

Pautas de consumo, 1500-1850

Bartolomé Yun y Jaume Torras

Sesiones simultáneas:

Instituciones financieras no bancarias

Andrés Hoyo y Gabriel Tortella

Educación, capital humano y desarrollo económico

Clara Eugenia Nuñez y David Sven Reher

Las cuentas de las regiones. La reconstrucción de series históricas de la contabilidad regional de España (siglos XIX y XX)

Santiago Zapata y Jordi Maluquer

Baldios, comunales, propios y arbitrios: propiedad, gestión y explotación (siglos XIV-XIX)

Sebastián Amarilla y Ángel García Sanz,

La pesca en la economía española (siglos XVIII-XX)

José Giráldez y Joám Carmona

La competitividad internacional de la empresa española

Carles Sudrià y Jesús M. ${ }^{a}$ Valdaliso

Los sistemas hidráulicos en la historia

Joaquín Melgarejo y Antonio Macías

Las migraciones a América

Blanca Sánchez Alonso y Vicente Pérez Moreda

Cambio tecnológico y transformación económica: indicadores y perspectivas

Santiago López y Patricio Sáiz 
Mujeres y hombres en los mercados de trabajo.

La evolución historica de los mecanismos de acceso $y$ movilidad interna

Carmen Sarasúa y Lina Gálvez

Sesión “Ernest Lluch" de Historia del Pensamiento Económico

Hacienda y Dinero en los economistas españoles Salvador Almenar y Vincent Llombart

Taller sobre las últimas investigaciones en historia del pensamiento económico en España

John Reeder

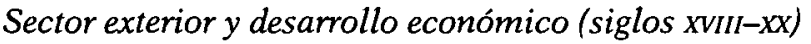

Josep M. ${ }^{\text {a }}$ Delgado y José M. ${ }^{\text {a }}$ Serrano Sanz

La formación y gestión de los grandes patrimonios

en España (siglos $X V-X X$ )

Hilario Casado y Ricardo Robledo

La automoción en la industralización española:

aspectos de demanda y oferta

José Luis Hernández Marco y José Luis García Ruiz

Sector agrario e industralización

Luis Germán Zubero y Antonio Parejo Barranco

\section{Información:}

Cuotas de inscripción:

Socios de la AHE: inscripción gratuita (A los socios que se inscriban antes del 21 de mayo de 2001 se les regalará el $C D$ con los textos presentados a las diferentes sesiones del congreso. A los que se inscriban después de esta fecha se les cobrará 3.000 pesetas por dicho $C D$ ).

Estudiantes: inscripción gratuita. Otros participantes: 15.000 ptas.

(A los participantes que se inscriban antes del 21 de mayo de 2001 se les regalará el $C D$ con los textos presentados a las diferentes sesiones del congreso. A los que se inscriban después de esta fecha se les cobrará 3.000 pesetas por dicho $C D$ ).

La inscripción da derecho a participar en todas las actividades programadas (visitas, excursiones, etc.)

Las cuotas se ingresarán mediante transferencia bancaria al siguiente número de cuenta: Ibercaja. Oficina Central Paraíso. Núm. de cuenta: 208501039003301070 19. Concepto: VII Congreso de la AHE

Inscripciones, reservas de alojamiento e información sobre el congreso: DI\&CO

Dinámica y Comunicación. Paseo Sagasta, 19. Entlo. dcha. 50008 Zaragoza.

Tel.: 976 233339. Fax: 976 212959. c-mail: ana@dico.es

Para más información: A través de la página web de la Universidad de Zaragoza: www.unizar.es/eueez/cahe/cahe.htlm o a través de la dirección de la Asociación de Historia Económica: www.adi.uam.es/ahe 


\section{EL TRIMESTRE $\mathrm{E}$

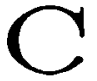 ONO NON

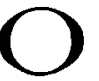

COMITÉ DICTAMINADOR: Alejandro Castañeda, Gonzalo Hernández Licona, Alejandro Werner. CONSEJO EDITORIAL: Edmar L. Bacha, José Blanco, Gerardo Bueno, Enrique Cárdenas, Arturo Fernández, Ricardo Firench-Davis, Enrique Florescano, Roberto Frenkel, Kevin B. Grier, Ricardo Hausmann, Alejandro Hernández, Albert O. Hirschman, Hugo A. Hopenhayn, David Ibarra, Folipe Larrain, Francisco Lopes, Guillermo Maldonado, Rodolfo Manuelli, José A. Ocampo, Joseph Ramos, Luis Ángel Rojo Duque, Gert Rosenthal, Francisco Sagasti, Jaime José Serra, Jesús Silva Herzog Flores, Osvaldo Sunkel, Carlos Tello, Sweder van Winjberger.

Director: Rodolfo de la Torre. Subdirector: Pablo Cloter Secretario de Redacción: Guillermo Escalante A.

\begin{tabular}{lll}
\hline Vol. LXVIII (2) México, Abril-Junio de 2001 & Núm. 270
\end{tabular}

Oscar Sánchez, Juan Seade y Alejandro Werner

Juan Carlos Morán Álvarez y Pedro Álvarez Martínez

Rubén Chavarín Rodriguez

María Eugenja Ibarrarán Viniegra y Roy Boyd

Fermando Toboso
Los costos asociados al proceso desinflacionario

Medida del desarrollo humano para los paises de la América Latina

El costo del desempleo medido en producto. Una revisión empirica de la ley de Okun para México

Los impuestos al carbono y la economia mexicana. El efecto del cumplimiento de las restricciones impuestas por el calentamiento mundial. El caso de México

Un primer análisis cuantitativo de la organización teritorial de las tareas de gobiemo en España, Alemania y Suiza

EL TRIMESTRE ECONóMico aparece en los meses de enero, abril, julio y octubre. La suscripción en Méxlco cuesta $\$ 180.00$. Número suelto $\$ 60.00$. Número suelto atrasado $\$ 40.00$. Disquetes con el índice general (por autores y temático) de los números 1-244, $\$ 26.00$ (4.49 dls.)

Precios para otros paises (dólares)

\begin{tabular}{lccc} 
& Suscripciones & \multicolumn{2}{c}{ Números sueltos } \\
\cline { 3 - 4 } & & Del año & Atrasados \\
Centroamérica y el Caribe & 70.00 & 20.00 & 10.00 \\
$\begin{array}{l}\text { Sudamérica y Esparia } \\
\begin{array}{l}\text { Canadá, Estados Unidos y resto del } \\
\text { mundo }\end{array}\end{array}$ & 90.00 & 30.00 & 20.00 \\
\hline
\end{tabular}

Fondo de Cultura Económica, carretera Picacho Ajusco 227, Col. Bosques del Pedregal, 14200 México, Distrito Federal. Suscripciones y anuncios: teléfono 522746 70, señora Irma Barrón. Correo electrónico (E-mail): trlmestre@fce.com.mx

Página del Fondo de Cultura Económica en Internet: http://www.fee.com.mx 
Gabriel Tortella (Previdente)

\section{PATRONATO}

Carmen Iglesias (Directora del Centro de Estudios Politicos y Constituclonales)

Carlos Pascual (Director de Marcial Pons)

Francisco Prada Gayoso (Director de la Pundacion Empreasa Pública)

Felipe Ruiz Martín (Preadente Honorario de la Asociación de Historia Económica) 


\section{Revista de Historia Económica}

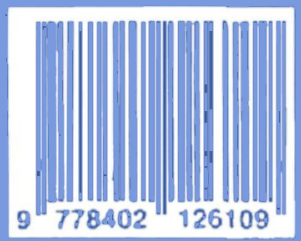

https://doi.org/10.1017/S0212610900009496 Published online by Cambridge University Press 